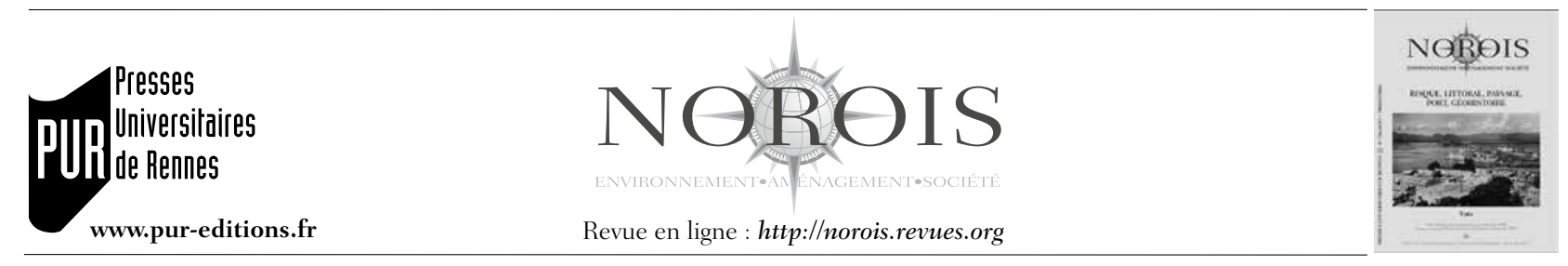

\title{
Note de recherche \\ Contribution à la mise en place d'indicateurs de suivi de la gestion des risques côtiers en France Métropolitaine : vers un observatoire intégré des risques d'érosion submersion \\ Contribution to the Implementation of Indicators for Monitoring Coastal Risk Management in Metropolitan France: Towards an Integrated Observatory of Seeflooding Erosion Risks
}

\author{
Étienne Quilleta ${ }^{a}$, Catherine Meur-F́́nEc ${ }^{b}$, \\ Étienne Chauveau c et Manuelle Philippe ${ }^{\mathrm{d}}$
}

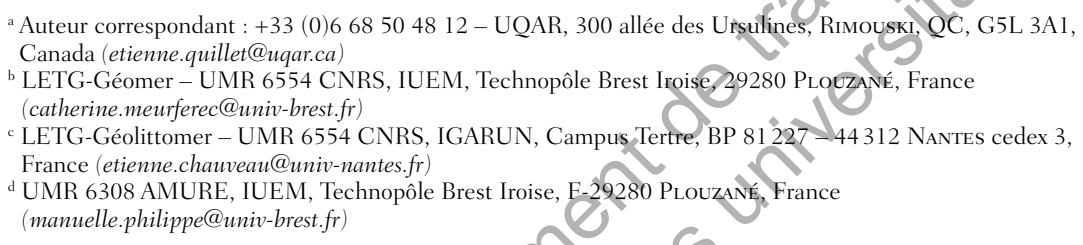

Résumé : La zone côtière offre un point de contact entre une nature potentiellement destructrice et une société exposée. Cela est visible par la survenue de dommages liée à Y'érosion côtière et la submersion marine, rendant le territoire vulnérable et la gestion de ces risques impérative. À partir d’une approche systémique de la vulnérabilité (vu comme la combinaison des aléas, des enjeux, de la gestion et des représentations), cette contribution détaille la méthode appliquée pour aboutir à une série d'indicateurs de suivi de la gestion des risques côtiers (érosion et submersion marine) en France métropolitaine. Ce travail rentre dans le cadre du projet de recherche OSIRISC, financé par la Fondation de France, qui vise, à terme, à construire un observatoire intégré de la vulnérabilité aux risques d'érosion-submersion.

\begin{abstract}
Coastal area is a contact point between hazardous nature and sensitive society, where coastal erosion and marine flooding risks are making the territory vulnerable and the risk management necessary. This contribution draws on systemic vulnerability approach (combination between hazard, issue, management and representation), and it details the indicators development method to monitor the coastal risk management in metropolitan France. This study incorporates to the OSIRISC research project. It aims to build an integrated observatory of vulnerability about coastal risks.
\end{abstract}

Mots clés : Gestion des risques - Érosion côtière - Submersion marine - Vulnérabilité - Indicateur

Keywords: Risk Management - Coastal Erosion - Marine Flooding - Vulnerability - Indicator 


\section{INTRODUCTION}

Le projet OSIRISC (ObServatoire Intégré des RISques Côtiers d'érosion et submersion), soutenu par la Fondation de France, a pour objectif le suivi de la vulnérabilité des territoires soumis à ces aléas. À terme, il vise à offrir des outils et une méthode pour permettre la mise en place d'un observatoire opérationnel intégré sur les territoires côtiers. Plus largement, le projet apparait comme un dispositif d'appui à la recherche et d'aide à la décision par l'apport de connaissances sur l'évolution de la vulnérabilité des territoires côtiers. Cette double finalité fait lien avec le principe d'intégration entre scientifiques et gestionnaires, propre au processus de GIZC (Miossec, 2012). En matière de risques côtiers, il a été montré la nécessité d'une approche pluridisciplinaire qui « offre une vision élargie des problématiques du littoral »(Meur-Férec, 2006). Ce projet y répond en associant quatre laboratoires de géographie, de droit et économie, de géoscience et de psychologie. Cette pluridisciplinarité (Chaumillon et al., 2017) permet d'appréhender la vulnérabilité comme un système.

Quatre composantes sont définies dans l'approche systémique de la vulnérabilité : les aléas, les enjeux, la gestion et les représentations (Hellequin et al. 2013; Henaff et al., 2014; Meur-Férec, 2006; Meur-Férec et al., 2008), et chacune d'entre elles fait l'objet d'un groupe de travail au sein du projet OSIRISC. Si l'évolution des aléds, et dans une moindre mesure des enjeux, sont très étudiées en France (CETMEF, 2009; GIP Littoral Aquitain, 2012 ; Chevillot-Miot et Mercier, 2014; Daubord, 2014 ; Bernon et al., 20 (6), il n'en est pas de même pour la gestion (au croisement de la géographie et du droit) et les représentations (qu'étudie la psychologie sociale) autour des risques côtiers ${ }^{1}$. Le suivi de ces deux composantes est plus innovant. Pourtant, la gestion et les représentations constituent les principaux leviers d'action pour réduire la vulnérabilité aux risques côtiers.

C'est la composante gestion qui fait plus spécifiquement l'objet de cette contribution. La gestion des risques côtiers s'entend ici à la fois par la façon dont sont appliquées les politiques publiques de

1. Depuis quelques années, plusieurs travaux intègrent déjà la notion de représentation du risque, comme les ANR Miseeva, Cocorisco et Ricochet, les projets EVADRisk et SHORELINE, les publications de H. ReyValette et al. (2015), E. Michel-Guillou et C. Meur-Férec (2016). prévention, de protection et de gestion de crise, et aussi par les stratégies et initiatives locales de réduction de la vulnérabilité. L'état de l'art réalisé par S. Lendre (2016), cherchant à recenser les différents moyens de suivre dans le temps l'effet de la gestion sur la vulnérabilité, première étape du projet OSIRISC, fait le constat d'une relative inexistence d'études et d'outils répondant à cette question, notamment à l'échelle locale. Pourtant, la gestion révèle l'état des politiques publiques et l'avancée de leurs applications sur les territoires côtiers, influençant les représentations des habitants, l'extension des enjeux et l'impact des aléas. Il est donc primordial de suivre cette composante gestion. Cet article présente la méthode employée pour obtenir une série d'indicateurs de suivi deda composante gestion de la vulnérabilité systémiquẹe.

\section{SUIVRE LA GESTION POUR RÉDUIRE LA VULNÊRABILITÉ}

\section{Approche systémique de la vulnérabilité}

¿a vulnérabilité est aujourd'hui un concept centrál en géographie et sa définition, qui reste polysémique, est de plus en plus précisée, supportée par un corpus théorique et méthodologique important (Leone, 2007). Souvent, on distingue la vulnérabilité physique, soit les impacts physiques (quantifiables) d'un aléa sur un enjeu, de la vulnérabilité sociale, soit la sensibilité de la société face aux aléas (D’Ercole, 1996; Brooks, 2003; Leone et Vinet, 2006; Reghezza, 2006). La vulnérabilité physique peut être définie comme " l'impact physique d'un aléa sur des bâtiments, des réseaux, des infrastructures, des populations »(Reghezza, 2006). Cette vision de la vulnérabilité soutient une capacité à mesurer les dommages subis (en nombre de décès suite à un événement par exemple).

À l'inverse, la vulnérabilité sociale aborde ce concept d'un point de vue humain, partant du constat qu'à probabilité d'occurrence d'un aléa constante, c'est la vulnérabilité de la population qui aboutit à une catastrophe plus ou moins importante (Wisner et al., 1976). Elle s'inspire de la géographie anglosaxonne des années 60 et 70, l'école de Chicago, incarnée notamment par G.F. White et B. Wisner, qui placent la société au cœur de l'étude des risques, au travers de sa capacité d'adaptation 
(adaptive capacity) et de réponse (coping capacity) face aux aléas (Reghezza, 2006). Ainsi, R. D'Ercole l'a défini comme la «propension d'une société donnée à subir des atteintes ou dommages en cas de manifestation d'un phénomène naturel, anthropique ou mixte » (1994). F. Leone (2007) conforte cette définition en considérant la vulnérabilité comme la « propension à l'endommagement ou au dysfonctionnement de différents éléments exposés (biens, personnes, activités, fonctions, systèmes) constitutifs d'un territoire et d'une société donnés ».

Face à cela, nous appréhendons ce concept par une approche globale, comme « un système, articulé autour d'un grand nombre de variables, naturelles et humaines, dont la dynamique dans le temps et dans l'espace peut engendrer des situations plus ou moins dangereuses pour une société exposée » (D’Ercole, 1998). F. Leone et F. Vinet (2006) insistent sur les interactions complexes entre les systèmes territoriaux, sociaux, institutionnels et environnementaux (ou naturels). Cette approche intègre pleinement une dimension sociale à la vulnérabilité, et fait lien entre une société et son environnement, objet d'étude de la géographie (Metzger et D'Ercole, 2011).

Cette vision systémique a été approfondíe dès 2004, dans le cadre de plusieurs projets de recherche, à commencer par le Programme National Environnement Côtier (PNEC, 2002-2004) (MeurFérec et al., 2004), puis de l'ANRMiseevá(20012011 ) et, plus récemment, lors de l'ANR Cocorisco (2011-2014) (Hénaff et al. 2014). La vưunérabilité globale ou systémique, défendue dans l'ensemble de ces projets, intègre, à la définition classique des risques côtiers (aléas et enjeuxx), les mesures de gestion de ces risques et leurs représentations par la population et les acteurs concernés. La vulnérabilité résulte donc « de la combinaison de tous ces facteurs, les aléas, les enjeux qui y sont exposés, les pratiques de gestion mises en œuvre [ou non] [...], ainsi que les représentations que s'en font les usagers »(Henaff, 2014). L’analyse de la vulnérabilité systémique doit donc considérer ces quatre composantes (figure 1). Les aléas et les enjeux forment les risques d'érosion et de submersion marine. La gestion s'apparente aux outils et mesures de prévention et de protection établis par les autorités compétentes. Les représentations font appel aux perceptions des risques côtiers par la population et aux comportements qui en découlent (Meur-Férec et al., 2008; Hellequin et al., 2013; Henaff et al., 2014).

La mise en œuvre d'une gestion des risques côtiers vise à prévenir les aléas, maitriser les enjeux et favoriser la prise en compte du risque par la société civile. Même si des erreurs peuvent être faites, nous partons du principe que toutes actions de gestion visent à répondre à un impératif de réduction de la vulnérabilité, Et aux vues des nombreux outils et stratégies de gestion apparus ces dernières années, cette composante a un rôle majeur au sein de la vulnérabilité systémique. C'est pour suivre l'évolution dela gestion des risques côtiers, et donc les leviers de réduction de la vulnérabilité, qu'un outil permettant son suivi est proposé.

\section{L'indicateur, un outil de simplification en réponse à la complexité}

Parmi les différents outils d'évaluation qui existent, notre recherche se concentre tout particulièrement sur la mise en œuvre d'indicateurs. Les indicateurs, quantitatifs ou qualitatifs, sont très lar-
Figure 1 : Représentation de la vulnérabilité systémique / Source : Meur-Férec C., 2006

sentation

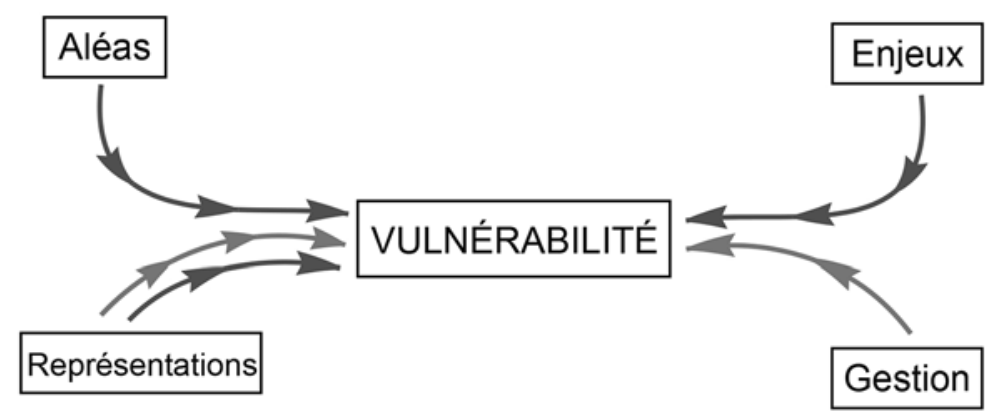

accroissement de la vulnérabilité $\quad$ diminution de la vulnérabilité 
gement utilisés dans le domaine de l'évaluation des politiques publiques, dans un objectif de développement durable (Gallopin, 1997), et plus spécifiquement, de gestion intégrée des zones côtières (Laffite et al., 2012). Par exemple, le consortium européen DEDUCE a mis en avant « le besoin de développer des indicateurs pour évaluer la performance (l'exécution) des démarches de GIZC » (DEDUCE Consortium, 2006).

L'utilisation des indicateurs présente un intérêt grandissant pour l'évaluation des politiques publiques littorales, mais leur diversité rend leur définition très large. G.-C. Gallopin (1997) rappelle qu'un indicateur peut effectivement être : «une variable, un proxy, une mesure $[. .$.$] , une valeur, un$ compteur, une fraction, etc. ». Il est donc impératif de construire des indicateurs dont les caractéristiques répondent à notre objectif : suivre la gestion des risques côtiers et son effet sur l'évolution de la vulnérabilité. Ainsi, nos indicateurs présentent plusieurs caractéristiques.

Tout d'abord, les indicateurs doivent être une « information finalisée ou instrumentale servant caractériser une situation évolutive [...], de façon a les évaluer et à les comparer en leur état à d’autres dates passées ou projetées »(Plan bleu, 2006 in Dupont, 2016). L'information de départ est doné analysée, triée et simplifiée pour que le résultat puisse être communiqué et qu'une tendance puisse être perçue sur plusieurs annêes, permettant d'obtenir le suivi souhaité dans le cadre du projet. En réponse à la complexité de la gestion des risques côtiers, un indicateur est ensulite "sélectionné parmi un ensemble de variables pour son caractère représentatif au seind'un système complexe » (Dupont, 2016). Il devient une simplification de la réalité pour en faciliter la lecture et l'usage, impliquant de choisir les variables les plus représentatives parmi l'ensemble d'outils et concepts qui composent cette gestion des risques côtiers. En ce sens, les indicateurs n'affichent pas la réalité, mais ils fournissent une tendance sur l'état général d'un sujet. Ils ont donc un rôle de connaissance sur la gestion sur un territoire donné. Pour que le suivi puisse avoir lieu, les indicateurs doivent pouvoir être renseignés régulièrement. Étant donnée la double finalité du projet, l'apport de connaissance et l'aide à la prise de décision, leur méthode de renseignement doit être reproductible par des chercheurs ou des gestionnaires (chargés d'études ou de missions aux « risques côtiers » des différentes structures publiques locales, régionales et nationales). Cela implique un accès aux données et des compétences adaptées aux capacités techniques, financières, temporelles de chaque utilisateur.

La construction d'indicateurs pour suivre la vulnérabilité est donc justifiée en plusieurs points. Tout d'abord, l'outil « indicateur » permet le suivi de l'évolution temporelle de la vulnérabilité d'un territoire, ou la comparaison entre les territoires, selon des critères standardisés. Ensuite, la mise en place d'une série d'indicateurs vise à faire avancer la connaissance scientifique et à appuyer les gestionnaires dans leur gestion des risques côtiers. La collecte de séries de données effectueé dans le cadre du renseignement des indicateurs et, qui plus est, mise à disposition en ligne, est un support de recherche intéressant. Ehfin, la simplification apportée par ce mode de mesure permet d'appréhender de façon synthétique la complexité de la gestion des risques côtiersen France. La conservation des données brutes permet une lecture plus détaillée des indicateurs, pour remédier à une approche partielle de la gestion.

\section{Une gestion complexe des risques côtiers en France}

Par gestion du risque, nous entendons « l'ensemble des étapes, organisations et mesures structurelles et non structurelles, plus ou moins coordonnées entre elles, que les sociétés mettent en place pour lutter contre les risques » (Veyret, 2007). Le contexte des vingt dernières années implique la nécessité de gérer les risques d'érosion - submersion. Les dommages humains et financiers qu'ils engendrent pèsent sur la société; par exemple, la tempête Xynthia de 2010 a fait 47 victimes et coûté 1,5 milliards d'euros d'indemnisation (Chauveau et al., 2011). De plus, le développement durable dans lequel nous nous inscrivons vise à établir une vision à long terme de la gestion des territoires. Sur le littoral, le processus de GIZC, qui puise son origine dans le concept de développement durable, met donc en avant des principes autour du rapport Nature - Société qui sont au cœur de l'approche géographique. Comme l'explique Rey-Valette et al. à propos de la gestion du risque d'érosion en Méditerranée, « l'évolution 
actuelle des politiques de gestion de l'érosion, quant à la prise en compte des acteurs, s'inscrit parfaitement dans les principes d'intégration et de concertation prônés par la GIZC » (2006).

Les acteurs ayant les compétences pour gérer les risques côtiers sont nombreux et leurs interactions complexes (Deboubt, 2010). Cette complexité est notamment soulignée par « le contraste entre les stratégies de développement au niveau global [...], et la réalité des situations sur le terrain » (MeurFérec et Rabuteau, 2014). Le maire, ayant autorité de police, se doit « d'assurer le bon ordre, la sureté, la sécurité et la salubrité publique ». Il lui revient « le soin de prévenir [...] et de faire cesser [...] les inondations, les ruptures de digues, les éboulements de terres, $[\ldots]$ ou tout autre accident naturel ${ }^{2}$ ». Ainsi, les collectivités locales, et les établissements publics ayant reçu cette compétence, ont obligation de prévenir et protéger les populations des risques côtiers. Parallèlement dans une société « d'Etat providence », ce dernier se doit d'offrir aux collectivités territoriales les outils et moyens nécessaires pour gérer ces risques. Effectivement, les élus locaux soulignent que « l'intervention de ce dernier [l'Etat] permettrait de pallier les difficultés et de mobiliser les financements et les acteurs nécessaires" " (Minéo-Kleiner, 2017). Les différents ministères et les services déconcentrés de l'Etat cherchent donc à définir des stratégies de gestion et assister les collectivités locales dans la constructioncde leur prévention des risques, par l'élaboration de textes juridiques, de plans d'action et d'appels â projet.

Très concrètement, cefte gestion se construit principalement au travers de deux logiques distinctes. La première met en avant la prinauté de l'État établissant des préconisations, qui devront être appliquées à l'échelle locale, passant par la mise en œuvre d'outil de gestion spécifique sur un territoire. Deux stratégies issues d'une logique descendante abordent les risques côtiers de manière dissociée : la stratégie nationale de gestion des risques d'inondation (SNGRI) et la stratégie nationale de gestion intégrée du trait de côte (SNGITC). Elles fixent chacune des préconisations et un plan d'action à l'échelle nationale visant à orienter les politiques publiques aux niveaux régional et local. Par ailleurs, l'Etat a identifié, après Xynthia, des territoires prioritaires ayant obligation de respecter

2. L2212-2 du Code Général des Collectivités Territoriales, 9/06/2017 un PPRL (circulaire du 2 août 2011). Le PPRL, à partir d'un événement modélisé par une cote de référence, contraint le Plan Local d'Urbanisme (PLU) en établissant, sur le territoire, différents zonages d'inconstructibilité et différentes prescriptions sur les constructions existantes et futures (DGPR, 2014). De plus, il impose la mise en œuvre d'un PCS (Plan Communal de Sauvegarde) sur le territoire concerné, document de gestion de crise détaillant le processus organisationnel à suivre en cas d'événement dommageable majeur.

Parallèlement, dans une logique de décentralisation croissante, certaines compétences sont attribuées aux collectivités locales et la mise en œuvre de certains outils s'effectue à l'initiative de ces mêmes collectivités. Issus de cette logique ascendante, les collectivités territoriales ont eu la possibilité de répondre aux appels à projet PAPI en 2003, 2012 et 2017. Ce plan d'action, financé en partie par le fonds national de prévention des risques naturels majeurs, dit Fonds Barnier, vise à « réduire les conséquences dommageables des inondations » (DGPR, 2017). De surcroit, le processus de décentralisation des compétences de l'Etat vers les collectivités, notamment par la Loi MAPTAM du 27 janvier 2014, attribue à ces dernières des compétences en Gestion des Milieux Aquatiques et Prévention des Inondations (GEMAPI). La GEMAPI a pour objectif, entre autres, de rassembler la gestion des ouvrages de protection à une seule autorité compétente, l'EPCI, à travers un système d'endiguement. Ces outils, bien que non spécifiques au phénomène de submersion marine, se doivent d'être pleinement cohérents avec la SNGITC et la SNGRI, et leurs applications régionales et locales (GIP Littoral Aquitain, 2012).

De nombreux éléments caractérisent ainsi la gestion des risques côtiers en France métropolitaine :

- des principes de gouvernance et d'intégration issus de la GIZC;

- deux types d'acteurs principaux, et deux échelles de gouvernance (Etat et Collectivités territoriales) dont les points de vue et les intérêts peuvent, par ailleurs, diverger;

- des compétences et des capacités d'action issues majoritairement d'une logique descendante pilotée par l'État, mais avec une forte tendance à la décentralisation, rendant les relations entre acteurs complexes, parfois conflictuelles; 
- une diversité d'outils, dont beaucoup se rapportent à un risque en particulier, pas toujours adaptés aux risques côtiers (inondation continentale par exemple), et une approche distincte entre les risques d'érosion et de submersion, avec des niveaux de contraintes plus ou moins forts et des échelles d'application variables du local au régional.

L’ensemble de ces éléments aboutit à une gestion des risques d'érosion - submersion en France métropolitaine éminemment complexe. Réduire cette complexité à quelques indicateurs entraine une perte inévitable d'information, mais permet de suivre la mise en place de ces politiques publiques, qui peut différer fortement d'un territoire à l'autre. Pour autant, cette simplification ne doit pas déboucher sur une vision simpliste, donnant une image erronée de la réalité de la gestion des risques côtiers. Les indicateurs ne vont pas pouvoir évaluer l'intégralité de ce qui compose la gestion, et ce n'est pas leur rôle. Mais ils doivent, avant tout, mettre en avant des tendances et des situations générales qui font avancer la réflexion autour de cette gestion, et plus largement autour de la réduction de la vulnérabilité. Il est donc nécessaire d'effectuer des choix parmi l'ensemble des éléments qui caractérisent la gestion des risques côtiers, pour déboucher sur des indicateurs représentatifs dont le renseignement régulier permettra d'appréhender l'évolution.

\section{MÉTHOde DE CONSTRUCTION}

\section{DES INDICATEURS DE SUIVD DE}

\section{LA GESTION DES BISQUES CÔTIERS}

\section{Cadre théorique et méthodologique à la construction d'indicateurs}

Pour construire ces indicateurs de suivi de la gestion des risques côtiers, nous pouvons nous appuyer sur la méthode avancée par P.-F. Lazarsfeld (1958), reprise par P.-M. Boulanger (2004). Ces derniers identifient différentes étapes théoriques de construction d'indicateurs. Dans un premier temps, le « concept » étudié est décomposé en différentes "dimensions », soit les facettes et aspects significatifs du concept. Ensuite, des éléments mesurables au sein de ces "dimensions " sont retenus en tant que "variables ». Ces dernières deviennent des «indicateurs " lorsqu'elles sont associées à des méthodes de collecte de l'information formalisées.
L'objet de cette contribution traite uniquement de la construction d'" indicateurs » à partir des différentes "dimensions" de la gestion des risques côtiers, proposée par cette méthode (partie en noire sur la figure 2). En amont de notre travail, l'analyse $\mathrm{du}$ «concept» (ici le dispositif théorique de gestion) a débouché sur une série de «dimensions » (ici les outils et démarches formelles et informelles qui concrétisent la gestion des risques côtiers en France métropolitaine). En aval, l'éventuelle pondération et l'agrégation des «indicateurs » retenus feront l'objet d'une phase ultérieure du projet OSIRISC, dont l'objectif est d'élaborer un «indice » global de vulnérabilité systémique (associant les composantes Aléas, Enjeux, Gestion et Représentations).

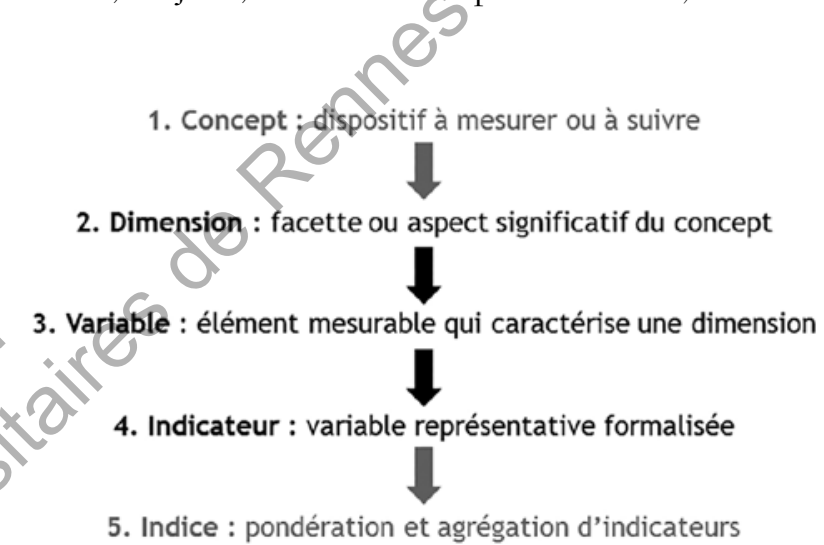

Figure 2 : Méthode théorique à l'élaboration d'indicateurs, d'après Boulanger P.M., 2004

Theoric method for indicators development, inspired by Boulanger P.M., 2004

Ainsi, il s'agit ici d'élaborer des indicateurs de gestion. Pour ce faire, « les différentes dimensions [du concept] sont découpées en variables dont certaines sont retenues au titre d'indicateurs " (Boulanger, 2004). Il est donc nécessaire d'identifier et de sélectionner ces variables («indicateurs potentiels»), puis d'établir leurs caractéristiques (tels que le protocole de renseignement, les échelles spatiales et temporelles) à travers des fiches techniques, afin d'en faire des «indicateurs opérationnels». Pour cela, nous établissons une méthode en quatre étapes opérationnelles qui, à partir des «dimensions » de la gestion, nous font cheminer vers les indicateurs de suivi de la gestion des risques côtiers en France métropolitaine (figure 3). 


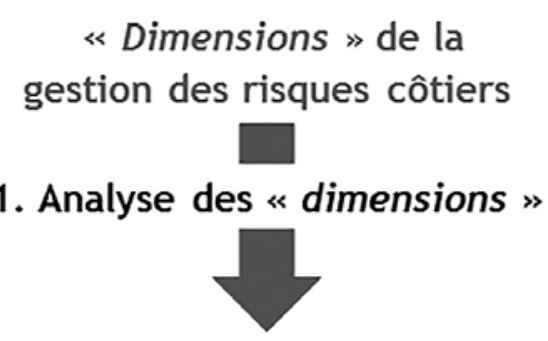

«Indicateurs potentiels» 1

2. Consultation auprès de gestionnaires

1

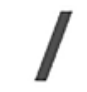

\section{Evaluation Faisabilité- Qualité scientifique 1}

4. Evaluation Faisabilité-Utilité

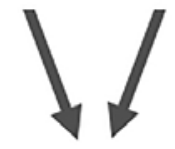

«Indicateurs opérationnels »

Figure 3 : Méthode de construction d'indicateurs de suivi de la gestion des risques côtiers (Source : Quillet E., 2017) ment monitoring

Method to develop indicators for coastal risks manage-

\section{Des leviers potentiels de réduction de la vulnérabilité}

L'objectif de cette premiere étapelest donc d'établir une liste d'indicateurs potentiellement intéressants, en analysant le contenu et les objectifs de chacun des outils (PPRL, GEMAPI, PAPI, Stratégies nationales par exemple), l'application des principes de GIZC (gouvernance, intégration, etc.) et les compétences des acteurs (État et collectivités) qui participent à la gestion des risques côtiers. Pour ce faire, nous nous appuyons sur des documents de gestion ("PPRL Ouest Odet ", DDTM 29, 2016; « Stratégie Régionale de Gestion du Trait de Côte », GIP Littoral Aquitain, 2012; "PAPI du polder Combrit - Sainte Marine - Ile Tudy ", SIVOM Combrit - île Tudy, 2011 ; etc.), des guides méthodologiques d'aide à la construction d'outils de gestion (" Guide méthodologique Plan de prévention des risques littoraux », DGPR,
2014; « Cahier des charges PAPI 3 », DGPR, 2017; etc.), des études et rapports destinés à l'apport de connaissance (" La tempête Xynthia du 28 février 2010, retour d'expérience en Loire Atlantique et en Vendée », DREAL Pays de la Loire, 2012; «Submersion marine et érosion côtière : connaitre, prévenir et gérer les risques naturels littoraux sur la façade atlantique ", CESER de l'Atlantique, 2015; etc.), des publications scientifiques (« Analyse des dommages liés aux submersions marines et évaluation des coûts induits aux habitations à partir de données d'assurance », C. André, 2013; etc.), des textes de lois (Loi MAPTAM; Code de l'urbanisme; etc.) et des sites internet spécialisés (georisques.fr; irma-grenoble.com; etc.). Ainsi, à travers l'étude de ces différents documents, nous cherchons les «variables » liées à la gestion susceptibles d'influer sur la vulnérabilité systếmique.

L'identification des variables s'effectue selon trois critères. Ilss sinspirent des notions qui caractérisent un indicateur, à savoir la reproductibilité, la synthétisation du résultat et l'évolution temporelle. Cefte étape vise à ne conserver que celles qui pourrgnt être formalisées sous la forme d'indicateurs potentiels. Premièrement, il est nécessaire que les variables puissent refléter une tendance claire et univoque à l'augmentation ou à la diminution de la vulnérabilité systémique. Ensuite, le processus de renseignement de la variable se doit d'être formalisé et son résultat mesurable sur une échelle de 1 (faiblement réducteur de vulnérabilité) à 5 (fortement réducteur de vulnérabilité). Enfin, il est impératif que le résultat évolue dans le temps, c'est à dire qu'il ne soit pas fixe et absolu. Pour illustrer notre propos, la variable «ancienneté de révision $d u$ $P C S$ » répond aux trois critères car elle affiche une tendance unique d'augmentation de la vulnérabilité selon l'ancienneté de la révision, elle est formalisée par une unité de temps, elle se mesure en nombre d'années depuis la dernière révision, et elle évolue dans le temps en fonction d'une révision régulière ou inexistante du document. A l'inverse, la variable «présence d'un enrochement » ne peut pas aboutir à un indicateur potentiel. Elle ne traduit pas une tendance unidirectionnelle d'évolution de la vulnérabilité (un enrochement peut réduire ou augmenter l'aléa, selon sa construction, son impact environnemental et sa dégradation). Les deux types de réponses de cette variable (présence ou absence) 
ne permettent pas un calibrage progressif vers plus ou moins de vulnérabilité. Une fois qu'un enrochement est construit, la variable évolue peu durant plusieurs années.

Dans un souci de clarté, également dans l'optique d'aboutir à un indice global de vulnérabilité, nous avons structuré les indicateurs selon une arborescence. Celle-ci nous permet de regrouper des indicateurs définissant des "objets », eux même regroupés en «dimensions » selon les informations qu'ils fournissent. Cette première étape d'analyse des «dimensions » de la gestion et l'arborescence des indicateurs nous permet d'obtenir une liste de quatre-vingt-six indicateurs potentiels de gestion, répartis en six «dimensions» : la recherche, les aménagements, la sensibilisation aux risques, la planification spatiale, la gestion de crise et les stratégies de gestion. Le tableau 1 qui suit reprend les quatre-vingt-six indicateurs potentiels identifiés lors de cette première étape.

Cette liste d'indicateurs potentiels représente l'état d'avancement des réflexions sur la construction d'indicateurs de suivi de la gestion des risques côtiers à un moment donné. Elle a évolué tout au long de notre démarche, en fonction de l'approfondissement de chacun des indicateurs, qui étaient plus ou moins pertinents pour notre objectif. Cette évolution des indicateurs et des dimensions et ce tri sont issus du travail collectif des chercheurs durant le projet, mais aussi de l'apparition récente

\begin{tabular}{|c|c|c|}
\hline Dimension & Objet & Indicateurs potentiels \\
\hline \multirow{2}{*}{ 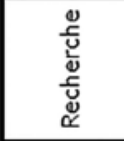 } & $\begin{array}{l}\text { Relation entre les sciences et } \\
\text { le territoire }\end{array}$ & $\begin{array}{l}\text { Implication dans la recherche scientifique / Ancienneté de l'observatoire OSIRISC / Publications scientifiques } \\
\text { traitant du risque sur le territoire }\end{array}$ \\
\hline & État des connaissances & $\begin{array}{l}\text { Accès à un centre de ressource communal / Dépôt des données sur une plateforme géographique / Fréquence } \\
\text { de mise à jour des données }\end{array}$ \\
\hline \multirow{2}{*}{ 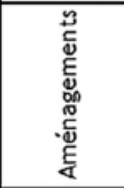 } & Ouvrages de protection & $\begin{array}{l}\text { Opposition aux dynamiques naturelles / Réversibilité / Cout d'entretien / Moyen financier prévu à la } \\
\text { construction / Efficacité de douvrage / Population à protéger }\end{array}$ \\
\hline & Mitigation & $\begin{array}{l}\text { Prescriptions sur le bâti existant / Prescription sur les futures constructions / Prescription sur les équipements } \\
\text { / Prescriptions et recommandations réalisées sur le bâti existant / Prescription et recommandations réalisées } \\
\text { sur les équipements }\end{array}$ \\
\hline \multirow{2}{*}{ 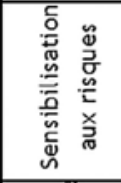 } & Implication de la & $\begin{array}{l}\text { Concertation population/décisionnaire / Plan familial de mise en sureté / Réserve communale de sécurité } \\
\text { civile (RCSC) CPrésence dune association sur les risques côtiers / Evènements associatifs sur les risques }\end{array}$ \\
\hline & Information à la population & $\begin{array}{l}\text { Risque dans les bulletins dinformations / Evènements publics de sensibilisation / Repères de crues / Réseau } \\
\text { de télealerte / Présence d'un DICRIM / Analyse pédagogique du DICRIM / Modes de diffusion du DICRIM / } \\
\text { Consultation du DICRIM }\end{array}$ \\
\hline \multirow{3}{*}{ 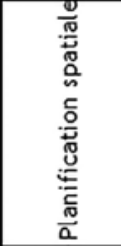 } & Domaine Public Maritime & Artificialisation du DPM / Occupation du DPM \\
\hline & $\begin{array}{l}\text { Schéma de Cohérence } \\
\text { Territoriale (SCoT) }\end{array}$ & $\begin{array}{l}\text { Caractère prospectif du document / Etat d'avancement de lélaboration du document / Actions de } \\
\text { concertation / Contentieux sur le SCOT / Distinction des risques côtiers pas rapport aux autres risques / } \\
\text { Intég ation des risques côtiers dans les différents documents / Prescriptions et préconisations sur les risques } \\
\text { côtiers / Indicateurs de suivi du SCoT sur les risques côtiers }\end{array}$ \\
\hline & & $\begin{array}{l}\text { Caractère prospectif du document / Etat d'avancement de lélaboration du document / Contentieux sur le } \\
\text { PLU / Réactualisation du document / Intégration des risques côtiers / Zonage du risque / Zonage Loi Littoral }\end{array}$ \\
\hline \multirow{2}{*}{ 总 } & $\begin{array}{l}\text { Plan Communal de Sauvegarde } \\
\text { (PCS) }\end{array}$ & $\begin{array}{l}\text { Présence d'un PCS / Ancienneté de révision du PCS / Acteurs ímpliqués dans le PCS / Présence de fiches } \\
\text { actions / Prise en compte des risques côtiers / Système d'alerte de la population / Cellule de crise / } \\
\text { Déclenchement du PCS / Formation des intervenants }\end{array}$ \\
\hline & $\begin{array}{l}\text { Organisation de la Réponse de } \\
\text { Sécurité Civile (ORSEC) }\end{array}$ & $\begin{array}{l}\text { Prise en compte des risques côtiers / Acteurs impliqués dans le dispositif / Déclenchement du disposotif / } \\
\text { Formation des intervenants }\end{array}$ \\
\hline \multirow{5}{*}{ 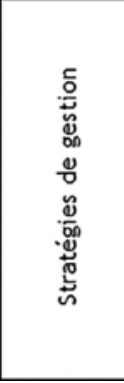 } & $\begin{array}{l}\text { Stratégie locale de gestion des } \\
\text { risques }\end{array}$ & $\begin{array}{l}\text { Présence de stratégies locales / Etat d'avancement de l'élaboration de la stratégie / Intégration sectorielle / } \\
\text { Intégration des stratégies nationales et régionales / Initiatives locales à lélaboration d'une stratégie / Scénarii } \\
\text { étudiés / Choix du mode de gestion }\end{array}$ \\
\hline & $\begin{array}{l}\text { Plan de Prévention des Risques } \\
\text { Naturels (PPRN) }\end{array}$ & $\begin{array}{l}\text { Présence de PPRN / Etat d'avancement de l'élaboration du PPRN / Contentieux sur le PPRN / Cote de de } \\
\text { référence choisie / Valorisation comme outil réglementaire / Zonage du PPRN }\end{array}$ \\
\hline & $\begin{array}{l}\text { Plan d'Actions de Prévention } \\
\text { des Inondations (PAPI) }\end{array}$ & $\begin{array}{l}\text { Présence d'un PAPI / Suivi du programme d'actions / Equilibre des dépenses entre les différents volets / } \\
\text { Concertation entre les acteurs }\end{array}$ \\
\hline & $\begin{array}{l}\text { Gestion des Milieux Aquatiques } \\
\text { et Prévention des Inondations } \\
\text { (GEMAPI) }\end{array}$ & $\begin{array}{l}\text { Cohérence avec la stratégie locale / Partenariats Autorité compétente - Propriétaire d'ouvrages / Niveau } \\
\text { d'eau considéré / Digues intégrées au système d'endiguement / Etat des digues / Zone protégée }\end{array}$ \\
\hline & Plan Submersion & dépenses / Initiati \\
\hline
\end{tabular}

Tableau 1 : Indicateurs potentiels de suivi de la gestion des risques côtiers, selon une arborescence Dimension - Objet - Indicateurs / Source : Quillet E., 2017

Potential indicators to monitor the coastal risks management according to a tree Dimension-Object-Indicator representation 
de certains outils et politiques en matière de gestion des risques côtiers. Par exemple, au cours de notre travail, le Plan Submersion Rapide (PSR) a été abandonné par l'État. Les indicateurs associés ont donc été retirés. A l'inverse, un indicateur relatif à la compétence GEMAPI a été élaboré suite à la mise en place de la règlementation.

\section{Une réflexion partagée pour identifier des principes de gestion}

L'objectif de cette étape est d'identifier les indicateurs les plus pertinents, en les confrontant à la pratique de la gestion des risques côtiers. Pour cela, les indicateurs potentiels ont été soumis, par thématique et au cours de cinq entretiens semi-directifs, à des chargés de mission au sein de collectivités locales bretonnes et vendéennes (Combrit, Lorient, Morlaix, Santec et Noirmoutier) confrontées aux risques côtiers d'érosion et/ou de submersion marine. Ces gestionnaires ont signalé leur intérêt pour le projet et sont, pour la plupart, membres du comité de suivi OSIRISC. Ils appliquent ou possèdent des compétences spécifiques en gestion des risques côtiers, et se distinguent donc des autres membres du comité de suivi, par une implication plus importante dans la construction des indicateurs.

Un guide d'entretien est construit autour des six «dimensions » et de leurs « objets » identifiés précédemment (figure 4). Les questions posées aucours de ces entretiens visent à déterminer si, selon les personnes enquêtées, les indicâteurs proposés per- mettent de rendre compte de la vulnérabilité d'un territoire et de son évolution. Notamment, il s'agit de savoir s'ils renseignent sur une direction précise d'évolution de la vulnérabilité. Par exemple, on demande si les gestionnaires sont d'accord sur le fait que plus il y a de contraintes d'urbanisation, moins il y a d'enjeux qui sont soumis à l'aléa, et plus la vulnérabilité diminue. Par ailleurs, on demande s'ils identifient des manques ou de redondances parmi les indicateurs proposés, s'ils ont d'autres indicateurs ou éléments à suggérer, au contraire s'ils rejettent certains indicateurs, qu'ils jugent inutiles ou impossibles à renseigner. Des questions spécifiques par « objets » sont également posées afin de déterminer les éléments les plus intéressants à considérer par nos indicateurs. Par exemple, nous cherchons à savoir par quels moyens la compétence GEMAPI peut permettre de réduire la vulnérabilité, ou bien s'il est possible, voir utile, de mesurer la réversibilité d'un ouvrage-sur le trait de côte, où encore s'il est nécessaire d'evaluer l'état général du PLU et du Schémacde Cohérence Territoriale (SCoT) pour appréhendêr la vulnérabilité.

Lanalyse des propos des gestionnaires contribue à enrichir les réflexions sur la construction d'indicateurs de suivi de la gestion des risques côtiers. Elle s'inscrit dans un processus de co-construction pour assurer au mieux l'efficacité et l'opérationnalité des indicateurs. Dans l'ensemble, les entretiens menés ont confirmé l'exhaustivité de l'approche adoptée. Selon les gestionnaires, l'ensemble de nos «dimensions » et «objets » couvrent globalement la gestion

\begin{tabular}{|c|c|}
\hline $\begin{array}{l}\text { Pour chaque dimension } \\
\text { : Recherche } \\
\text { : } \text { Aménagement } \\
\text { : Planifilisation aux risques } \\
\text { : Gestion de crise } \\
\text { - Stratégies de gestion }\end{array}$ & $\begin{array}{l}\text { - Est-ce que les objets, les variables et les indicateurs contenus dans } \\
\text { cette dimension permettent de rendre compte d'un niveau de } \\
\text { vulnérabilité ? } \\
\text { - Est-ce que les indicateurs déterminent une réduction plus ou moins } \\
\text { forte de la vulnérabilité? }\end{array}$ \\
\hline $\begin{array}{l}\text { Pour chaque objet } \\
\text { : PCS } \\
\text { : PAPI } \\
\text { : PPRN } \\
\text { : Mitigation } \\
\text { : Etat des connaissances } \\
\text { : } \text { Implication de la population } \\
\text { : }\end{array}$ & $\begin{array}{l}\text { - De quelle manière cet objet peut permettre de réduire la } \\
\text { vulnérabilité ? } \\
\text { - Identifiez-vous des manques dans la manière d'aborder cet objet ? } \\
\text { - Est-ce que les indicateurs contenus dans cet objet répondent à vos } \\
\text { attentes en terme de suivi de la vulnérabilité ? Ou en sont-ils trop } \\
\text { éloignés? }\end{array}$ \\
\hline
\end{tabular}

Figure 4 : Représentation synthétique du guide d'entretien (source : E. Quillet, 2017) Synthetic representation of the interview guide 
des risques côtiers, prenant en compte la grande majorité des leviers qu'ils peuvent utiliser pour réduire la vulnérabilité de leur territoire.

Néanmoins, les retours spécifiques sur chaque indicateur sont beaucoup plus nuancés, certains éléments pouvant faire apparaitre des points de vue divergents. Par exemple, certains gestionnaires pensent que la présence d'une digue réduit la vulnérabilité du territoire, car l'ouvrage protège des élévations du niveau marin. D'autres estiment que cet ouvrage augmente la vulnérabilité car il tend à intensifier les enjeux sur le territoire, que l'on croit protégé par la digue, alors qu'on ne peut exclure la probabilité que survienne un aléa qui dépasse les capacités de protection de l'ouvrage. De façon comparable, un indicateur sur la mise en ouvre d'exercice de déclenchement du Plan Communal de Sauvegarde (PCS), qui nous semble une variable importante de réduction de la vulnérabilité, suscite des commentaires mitigés. D'après certains gestionnaires, selon les acteurs qui y participent, la fréquence ou bien le type d'exercice proposé, ces exercices peuvent parfois être considérés comme contre productifs, créant des situations de «cri aux loups», de lassitude ou d'inquiétude, discréditant le dispositif auprès de la population, et finalement susceptible d'augmenter la vulnérabilité. Ces exemples mettent en avant les subtilités qui peuvent apparaitre lorsque l'on cherche à étudier un élément plus particulier. Il semble impossible de considerer l'ensemble des «variables» qui affichent ùne réduction de la vulnérabilité, tant leurs impacts surcecelle-ci peuvent être incertains. Cela renforce limpératif de simplifier le suivi de la gestion des risques côtiers, autour des indicateurs les plusclairs et les plus fiables. Plusieurs principes, établis à partir de nos lectures, des propos des gestionnaires et des réflexions qui en découlent, justifient la construction des indicateurs les plus pertinents.

Tout d'abord, les positions des acteurs à différentes échelles, du national au local, peuvent engendrer des situations conflictuelles, notamment lorsque des impératifs fixés par l'Etat dans une logique top-down sont difficilement appliqués ou applicables localement, ou lorsque des revendications locales sont difficilement compatibles avec l'intérêt général. Au sujet des PPRL, C. Perherin (2017) présente le fait que « l'Etat et les collectivités ne mettent pas en avant la même conception de la prévention : l'une s'appuyant sur une conception de gestion des cas de risque majeur engendré par l'aléa extrême et de réduction de la vulnérabilité et des dommages, l'autre s'appuyant sur une conception de maîtrise de l'aléa « ordinaire » par des ouvrages de défense ». Ainsi, le suivi des indicateurs sur l'état général du PPRL permet d'appréhender une mise en œuvre potentiellement délicate du document, par la prise en compte des contentieux portés au sujet des risques côtiers, également par la durée d'élaboration du plan. Cette articulation délicate des niveaux de gouvernance est également visible dans la construction des stratégies locales de gestion (érosion et submersion), qui doivent suivre les préconisations des stratégies nationales tout en s'appliquant aux spécificités des territoires et en intégrant les aspirations des acteurs locaux.

En outre, les entretiens avec les gestionnaires ont révélé que leurintégration dans un processus de gestion des risques côtiers a un impact important sur la réduction de vulnérabilité. Cette intégration peut scévaluer selon plusieurs approches. Au-delà de la diversité d'acteurs participant à une démarche de gestion, assez facile à estimer, il est possible de sintéresser à la mise en réseau du gestionnaire : soit le gestionnaire a l'occasion d'échanger avec ses collègues sur des problématiques de gestion, soit il reste plus isolé dans sa réflexion, ce qui contribue à accroitre la vulnérabilité. Pour certains " objets », il est possible de cibler un acteur fondamental et d'estimer son niveau de participation. Par exemple, comme suggéré lors des entretiens, le niveau d'intégration du Service Départemental d'Incendie et de Sécurité (SDIS) dans la mise en œuvre du PCS est important, car cet acteur possède des connaissances et des compétences essentielles, notamment dans l'organisation des premiers secours : cette implication du SDIS peut aller de l'absence d'intégration à un droit de modification du PCS.

Considérer la «gestion informelle » est souligné par les gestionnaires comme un élément à intégrer. Nous entendons par gestion informelle l'ensemble des démarches et décisions qui ne sont pas inscrites dans un schéma ou un dispositif institutionnel de gestion. Par exemple, la communication sur les risques côtiers, effectuée par des collectivités ou des associations, semble être une action déterminante pour renforcer la sensibilisation. Mais la diversité d'outils pour communiquer sur les risques côtiers 
rend difficile le suivi de l'ensemble des moyens de sensibilisation qui peuvent être mis en œuvre sur un territoire. Nous concentrons donc nos indicateurs sur les outils identifiés, au cours de nos entretiens, comme les plus pertinents. Ainsi, la présence de repères de crues et de reculs du trait de côte, ou bien le caractère pédagogique du Document d'Informations Communal sur les Risques Majeurs (DICRIM) font l'objet d'indicateurs.

Pour faire face à cette complexité et répondre à la faillibilité des indicateurs lorsqu'ils sont évalués séparément les uns des autres, il est indispensable d'avoir une approche systémique de ces derniers. En effet, un indicateur pris séparément ne rend pas compte de l'influence de "l'objet », et plus largement de la gestion des risques côtiers, sur la vulnérabilité. Dans une approche systémique, l'ensemble des éléments qui renseignent un «objet » offre une vision globale sur son état.

Cette étape indispensable de consultation des gestionnaires a permis de passer de plus de quatrevingts indicateurs à un système de vingt-sept indicateurs, en retirant les indicateurs potentiels les moins pertinents et en intégrant certaines suggestions des gestionnaires. A ce stade, ce nombre réduit rend possible l'intégration des indicateurs dans un observatoire opérationnel.

\section{ÉVALUER NOS INDICATEURS POUR RÉPONDRE AUX BESOINS DES GESTIONNAIRES ET DES CHERCHEURS}

Les deux dernières étapes de notre méthode visent à évaluer pour chaque indicafeur retenu, d'une part leur rapport faisabilité - qualité scientifique du point de vue des chercheurs membres du projet, et d'autre part leur rapport faisabilité - utilité du point de vue des gestionnaires. Ces deux évaluations cherchent à rendre les indicateurs opérationnels et à répondre à la double finalité du projet, à savoir l'apport de données pour la recherche scientifique, et l'aide à la prise de décision pour les élus et les gestionnaires.

Pour ces deux évaluations, trois critères ont été retenus : la faisabilité, la qualité scientifique et l'utilité. La faisabilité est jugée selon les moyens financiers, temporels et techniques à mettre en œuvre pour renseigner l'indicateur. Si le coût et les compétences techniques requises sont moindres, l'indica- teur sera facilement utilisable. À l'inverse, Si le coût et les compétences sont conséquents, l'indicateur sera difficilement utilisable, voire inutilisable dans le cas où les données et moyens nécessaires au renseignement de l'indicateur n'existent pas. Le critère de qualité scientifique (bonne, discutable ou mauvaise) est, quant à lui, établi selon la fiabilité (protocole rigoureux et précis) et la pertinence de l'indicateur (résultat influencé par des variables spécifiques aux risques côtiers). Si l'indicateur est influencé par des variables extérieures à notre thème d'étude et/ ou que son protocole de renseignement présente de nombreux biais, alors sa qualité scientifique sera jugée mauvaise, par les chercheurs, à dire d'expert. À l'inverse, si l'indicateur s'intéresse uniquement aux risques côtiers et que son protocole est rigoureux, sa qualité scientifique sera jugée bonne. Enfin, le critère d'utilité (indieateur jugé d'utilité mauvaise, discutable ou bonne) fait appel aux points de vue des gestionnaires, en lien avec leur approche de la vulnérabilité. Le gestionnaire jugera que l'indicateur est inutile sijlestime que celui-ci n'est pas pertinent/ intéressant pour étudier et suivre la vulnérabilité face aux risques côtiers, discutable s'il lui semble intéressant mais pas essentiel, où utile s'il répond à ses attentes en termes de suivi de la vulnérabilité. Il s'agit ici de l'expertise des gestionnaires membres du comité de suivi du projet OSIRISC, en tant que spécialistes de ces questions. Les chercheurs ayant travaillé sur toutes les façades françaises, le comité de suivi étant composé de gestionnaire de toute la France métropolitaine, cette étape confirme que les indicateurs peuvent être applicables sur l'ensemble du territoire national.

Dans un premier temps, ce sont les chercheurs membres du projet qui évaluent le rapport faisabilité - qualité scientifique des indicateurs (tableau 2). À la suite du test, ceux-ci sont conservés, modifiés, abandonnés, où bien gardés en réserve devant la probabilité que les compétences et les moyens permettent, un jour, de les utiliser. Ensuite, les gestionnaires jugent, selon le rapport faisabilité - utilité (tableau 2), les indicateurs les plus intéressants.

Ces deux évaluations s'inscrivent dans le processus de co-construction défendu par le projet. Elles ont abouti à la suppression et la modification de plusieurs indicateurs. Par exemple, la prise en compte des risques côtiers dans un Schéma de Cohérence Territorial n'a pas été conservée car son impact sur 


\begin{tabular}{|c|c|c|c|c|}
\hline & & & ment pour le & \\
\hline & & \multicolumn{3}{|c|}{ Qualité scientifique (uniquement pour les chercheurs) } \\
\hline & & Mauvaise & Discutable & Bonne \\
\hline \multirow{3}{*}{ Faisabilité } & $\begin{array}{l}\text { Degré de technicité } \\
\text { faible }\end{array}$ & Abandonné & Modifié & Conservé \\
\hline & $\begin{array}{c}\text { Degré de technicité } \\
\text { élevé }\end{array}$ & Abandonné & Modifié & Conservé \\
\hline & Infaisable & Abandonné & En réserve & En réserve \\
\hline
\end{tabular}

Tableau 2 : Evaluation des indicateurs selon un rapport Faisabilité - Qualité scientifique et Faisabilité - Utilité (source : Philippe M., 2017) Indicator assessment according to Feasability - Scientific quality and Feasability - Utility criteria

la vulnérabilité dépendait d'éléments extérieurs à notre objet d'étude (échelle d'application trop large et objectifs du SCoT non-centrés sur la gestion des risques par exemple); il n'était donc pas pertinent et ne répondait pas au critère de qualité scientifique. Dans un autre cas, l'indicateur sur la présence de contentieux dans l'élaboration du PPRL a été intégré à celui sur l'état général du document, afin d'aboutir à un indicateur global de suivi du PPRL. Finalement, sur les vingt-sept indicateurs évalués, sept sont retirés ou modifiés, pour aboutir à un système de vingt indicateurs.

\section{VINGT INDICATEURS DE SUIVI DE LA GESTION DES RISQUES CÔTIERS en France métropolitaine}

Toutes ces étapes constituant notre méthode, nous permettent d'abouttir à une grille d'indicateurs de suivi de la gestion des risques côtiers en France métropolitaine. Vingt indicateurs ont finalement été construits, et regroupés en cinq «dimensions»: Stratégie locale, Maitrise de l'urbanisation, Sensibilisation, Connaissance et Gestion de crise; chacun divisé en «objet » de suivi. Notre grille d'indicateurs conserve bien évidemment la structure «dimension - objet - indicateur» dans la perspective d'étudier par la suite la faisabilité d'un indice propre à chaque composante du projet, puis reflétant une vulnérabilité globale. Le tableau 3 suivant situe le positionnement des indicateurs de gestion finalement retenus les uns par rapport aux autres, et résume ce qu'ils cherchent à suivre.

La Stratégie locale fait appel à l'application des outils issus des politiques publiques de gestion des risques, centré sur la construction d'une stratégie locale (PAPI, SLGRI et SLGITC), l'intégration des acteurs, et la gestion du trait de côte. La démarche locale est appréhendée selon son état d'avancement, la prise en compte de la relocalisation comme solution à la réduction de la vulnérabilité et, si un PAPI est défin sur le territoire, selon les caractéristiques du programme d'actions. Concernant l'intégration des acteurs, celle-ci est appréhendée selon la diversité d'acteurs qui participent à la démarche locale, les moyens humains consacrés à la gestion des risques côtiers et la mise en réseau de ces gestionnaires. La gestion du trait de côte s'inscrit pleinement dans la mise en place de la compétence GEMAPI, en cours actuellement. Nous nous intéressons donc à cette mise en œuvre actuelle et à "l'état des ouvrages » qui seront gérés par la structure porteuse de la compétence.

Ensuite, le suivi de la Maitrise de l'urbanisation est centré sur la prévention des risques. Deux indicateurs constituent cette dimension : « l'état général du PPRL », considérant un grand nombre de variable sur ce document, et les «limites de constructibilité en zone d'aléa », superposant le zonage « aléa » du PPRL avec les zones constructibles du PLU. La majorité des indicateurs potentiels sur les documents de planification spatiale (PLU et SCoT) n'ont pas été retenus, notamment parce que ces outils ne traitent pas de la gestion des risques côtiers de manière centrale.

La Sensibilisation cible des actions engagées par la collectivité, dans le but d'informer, d'éduquer et de sensibiliser la population, face aux risques côtiers. Nous retrouvons :

- présence de « repères de crues et de reculs du trait de côte" ",

- présence d'associations sur ce thème, 
Indicateurs composante Gestion

\begin{tabular}{|c|c|c|c|}
\hline Dimension & Objet & Indicateur & Sous-indicateur \\
\hline \multirow{9}{*}{ Stratégie locale } & \multirow[t]{4}{*}{ Démarche locale } & \multicolumn{2}{|c|}{$\begin{array}{l}\text { Démarche locale de gestion des risques : cherche à savoir si la collectivité est active localement, } \\
\text { dans une démarche de gestion des risques côtiers, notamment par l'élaboration d'un PAPI et } \\
\text { d'une Stratégie Locale }\end{array}$} \\
\hline & & Etat de la stratégie locale & $\begin{array}{l}\text { Prise en compte de la relocalisation : évalue le suivi des } \\
\text { préconisations nationales et régionales en matière de gestion } \\
\text { des risques côtiers, par leur intégration dans la stratégie } \\
\text { locale }\end{array}$ \\
\hline & & \multirow[t]{2}{*}{ Etat du PAPI } & $\begin{array}{l}\text { Présence d'actions dans chaque axe : le PAPI considère-t-il } \\
\text { l'ensemble des sept axes qui le compose, par la présence } \\
\text { d'actions dans chacun de ces sept axes définis par le cahier } \\
\text { des charges? }\end{array}$ \\
\hline & & & \begin{tabular}{|l|} 
Mise en oeuvre du programme d'actions: analyse \\
I'application du programme d'actions du PAPI, en regardant le \\
décalage entre l'échéancier et l'état d'avancement des actions
\end{tabular} \\
\hline & \multirow[t]{3}{*}{$\begin{array}{l}\text { Intégration des } \\
\text { acteurs }\end{array}$} & \multicolumn{2}{|c|}{$\begin{array}{l}\text { Acteurs partenaires de la démarche locale : évalue le niveau d'intégration sectorielle de la } \\
\text { démarche locale, par la prise en compte de l'ensemble des acteurs concernés par les } \\
\text { problématiques de risques côtiers, dans la construction d'une gestiondes risques : qui participe } \\
\text { à la gestion des risques côtiers? }\end{array}$} \\
\hline & & \multicolumn{2}{|c|}{$\begin{array}{l}\text { Compétences humaines sur les risques côtiers : identifie les moyens humains que les collectivités } \\
\text { locales possèdent pour construire leur gestion des risques côtiers }\end{array}$} \\
\hline & & \multicolumn{2}{|c|}{$\begin{array}{l}\text { Intégration extraterritoriale : identifier le niveau d'intégration du territoire dans les réflexions } \\
\text { extraterritoriales sur les risques côtiers, appréhende la mise en réseau du gestionnaire, par sa } \\
\text { participation et son implication à des travaux sur le thème des risques côtiers }\end{array}$} \\
\hline & \multirow[t]{2}{*}{$\begin{array}{l}\text { Gestion du trait de } \\
\text { côte }\end{array}$} & \multicolumn{2}{|c|}{$\begin{array}{l}\text { Mise en place de la compétence : analyse l'état d'avancement dans la prise en charge de la } \\
\text { compétence GEMAPI par la collectivitée }\end{array}$} \\
\hline & & \multicolumn{2}{|c|}{$\begin{array}{l}\text { État des ouvrages : sult l'évolution de l'état des ouvrages qui composent le système } \\
\text { d'endiguement de la GEMAPI }\end{array}$} \\
\hline \multirow{2}{*}{$\begin{array}{l}\text { Maitrise de } \\
\text { l'urbanisation }\end{array}$} & \multirow[t]{2}{*}{$\begin{array}{l}\text { Prévention des } \\
\text { risques }\end{array}$} & \multicolumn{2}{|c|}{$\begin{array}{l}\text { Etat général du PPRL : évalue l'état général dans lequel se trouve le PPRL sur le territoire, à } \\
\text { travers son état d'avancement, la doctrine sur laquelle il s'appuie, son intégration dans le PLU et }\end{array}$} \\
\hline & & \multicolumn{2}{|c|}{$\begin{array}{l}\text { Limites de constructibilité en zone d'aléa : fait ressortir les secteurs ou les contraintes de } \\
\text { construction et d'aménagements ne sont pas suffisantes face à l'intensité de l'aléa }\end{array}$} \\
\hline \multirow{5}{*}{ Sensibilisation } & \multirow[t]{3}{*}{$\begin{array}{l}\text { Dynamique de culture } \\
\text { du risque }\end{array}$} & \multicolumn{2}{|c|}{$\begin{array}{l}\text { Reperes de crues et de reculs du trait de côte : analyse le nombre d'affichage des évènements de } \\
\text { submersion passés et du phénomène de recul du trait de côte en cours, par la présence de } \\
\text { repères signalés et expliqués sur le territoire }\end{array}$} \\
\hline & & \multicolumn{2}{|c|}{$\begin{array}{l}\text { Présence d'associations sur les risques côtiers : remarque l'implication de la population aux } \\
\text { problématiques concernant les risques côtiers, par la présence d'associations locales traitant } \\
\text { de cette thématique sur le territoire }\end{array}$} \\
\hline & & \multicolumn{2}{|c|}{$\begin{array}{l}\text { Évènements de sensibilisation dans les établissements scolaires : analyse les différents } \\
\text { évènements organisés pour sensibiliser les écoliers (primaire-collège-lycée) aux risques côtiers, } \\
\text { par la récurrence des évènements et le niveau d'implication des sensibilisés }\end{array}$} \\
\hline & \multirow[t]{2}{*}{ DICRIM } & \multicolumn{2}{|c|}{$\begin{array}{l}\text { Modes de diffusion du DICRIM : évalue l'impact du DICRIM sur la population, par la façon dont il } \\
\text { est diffusé }\end{array}$} \\
\hline & & \multicolumn{2}{|c|}{$\begin{array}{l}\text { Analyse pédagogique du DICRIM : détermine la qualité du DICRIM, par son caractère pédagogique } \\
\text { : les rappels historiques, la cartographie, l'origine des risques, etc. }\end{array}$} \\
\hline Connaissance & Connaissances & \multicolumn{2}{|c|}{$\begin{array}{l}\text { Publications scientifiques sur les risques côtiers : évalue l'intensité des travaux de recherches } \\
\text { relatifs aux risques côtiers sur un site déterminé, par le nombre de publications scientifiques }\end{array}$} \\
\hline \multirow{3}{*}{ Gestion de crise } & Démarche PCS & \multicolumn{2}{|c|}{$\begin{array}{l}\text { Intégration du SDIS dans le PCS : mesure le niveau d'intégration du SDIS (Service Départemental } \\
\text { d'Incendie et de Secours) dans le PCS (ou une démarche similaire au PCS), par son implication } \\
\text { dans la construction du plan }\end{array}$} \\
\hline & & \multicolumn{2}{|c|}{$\begin{array}{l}\text { Mise à jour du document : identifie la pertinence du PCS (ou d'une démarche similaire) par } \\
\text { rapport à la date de la dernière mise à jour des données recensées dans le document }\end{array}$} \\
\hline & Alerte à la population & \multicolumn{2}{|c|}{$\begin{array}{l}\text { Mode d'alerte à la population : identifie le ou les modes d'alerte utilisés par la collectivité pour } \\
\text { prévenir la population de l'approche d'une situation à risque }\end{array}$} \\
\hline
\end{tabular}

Tableau 3 : Indicateurs de suivi de la gestion des risques côtiers (source : Quillet E., 2017) Indicators to monitor the coastal risks management 
- actions de sensibilisation dans les établissements scolaires,

- DICRIM analysé selon des critères de diffusion et de pédagogie du document.

L'ensemble des indicateurs potentiels au sujet du DICRIM ont été assemblés au sein de ces deux derniers indicateurs. Nous cherchons ici à mesurer les actions entreprises par la collectivité pour sensibiliser les populations, et non pas les effets de ces actions sur la population.

La Connaissance est composée d'un indicateur sur le nombre de publications scientifiques sur un territoire donné, qui traitent des risques côtiers. Elle cherche à suivre l'implication des scientifiques sur ce territoire et détermine ainsi l'apport de connaissance, venant appuyer la gestion des risques côtiers.

Enfin, concernant la Gestion de crise, nous partons du principe que le SDIS est la structure ayant les compétences pour juger de la qualité d'un PCS adapté au territoire, en partenariat avec la collectivité. Face aux difficultés d'établir des critères de qualité de ce document, nous nous en remettons à l'implication de cet acteur, qui possède les compéz tences en matière de gestion des catastrophes, mais également une connaissance fine du territorre. De ce fait, son degré de participation dans l'élaboration et la mise à jour du PCS garantit une effieacité plus ou moins forte du plan. De cette manière, nous nous concentrons uniquement sur la mise en œuvre de ce dispositif, sans considérer la culture dar risque que peut susciter le PCS, notamment par Yintermédiaire d'exercices auprès de la population. Cette appropriation par la population, mais aussi par les gestionnaires, est intégrée à la composante Représentations du projet. Les « systèmes d'alerte à la population » doivent également faire ressortir différentes qualités de prévention à l'approche d'un phénomène naturel.

\section{Conclusion}

Notre démarche de construction d'indicateurs se fonde, dans un premier temps, sur une approche scientifique des politiques publiques de gestion des risques côtiers, s'appuyant sur des doctrines, des stratégies et des outils de gestion à diverses échelles, visant à réduire la vulnérabilité, ainsi que sur des travaux de recherche universitaire sur ce sujet. Dans un second temps, cette approche théorique, discutée entre gestionnaires et chercheurs spéciali- sés sur la gestion des risques côtiers, nous a permis d'identifier les indicateurs permettant d'indiquer les variables qui se rapprochent au mieux de la gestion opérationnelle, soit l'application concrète de ces indicateurs sur le terrain. Ainsi, d'un côté, les gestionnaires apportent un jugement sur leur faisabilité et leur utilité, validant un premier objectif d'aide à la prise de décision, défendu par le projet. De l'autre, les chercheurs évaluent les indicateurs selon leur faisabilité et leur qualité scientifique, afin qu'ils répondent au mieux au second objectif du projet, à savoir l'appui à la recherche.

Pourtant, malgré toutes ces étapes de construction, la représentativité des indicateurs et leur capacité à rendre compte de l'avancement d'une gestion durable réduisant $\mathrm{R}$ vulnérabilité continue à poser question. Dans un souci d'efficacité, nous aboutissons à uncnombre restreint d'indicateurs, faciles à renseigner, pour qu'ils soient accessibles aux chercheurs et gestionnaires. Nous ne cherchons pas à appréhender la gestion des risques côtiers de manière exhaustive, mais bien à suivre les éléments essentiels qui la composent.

Comme nous l'avons vu, la complexité de la gestion débouche sur une multitude d'outils, dont l'impact sur la vulnérabilité est variable. Au fur et à mesure de l'avancement de notre construction, nous nous apercevions que chaque indicateur posait de nouvelles questions pouvant être approfondies, et débouchant sur une problématique connexe. Cette complexité est apparue d'autant plus forte au vu des positions parfois divergentes entre les acteurs de la gestion. En effet, de nombreux facteurs peuvent rendre difficiles les échanges entre acteurs, en fonction des orientations nationales et de leurs applications locales, des intérêts généraux et particuliers, du degré de présence du risque sur un territoire, pas toujours corrélés à la sensibilité des parties prenantes, y compris de la société civile. Ainsi, de nombreux indicateurs s'intéressent aux relations entre acteurs et à leur capacité à échanger entre eux, sur la question des risques côtiers.

Au final, les vingt indicateurs de gestion construits cherchent à refléter une situation de la gestion des risques côtiers sur un territoire, et plus largement l'intégration de celle-ci dans un processus de gestion intégrée. Notre démarche s'appuie sur une logique d'interaction Chercheurs-Gestionnaires impérative à la réussite du projet, mais également au partage 
et à l'amélioration des connaissances sur ce thème. Les indicateurs sont construits à partir des outils et mesures qui composent, actuellement, la gestion des risques côtiers en France Métropolitaine. Ils devront donc être pérennisés en étant testés sur plusieurs territoires ${ }^{3}$ pour qu'ils s'améliorent et s'adaptent à l'évolution de cette gestion, comme l'application de la compétence GEMAPI.

À la suite de ce travail, le projet OSIRISC va être confronté à deux étapes majeures en vue d'aboutir à un observatoire opérationnel. Tout d'abord, comment relier les quatre composantes Aléa - Enjeu - Gestion - Représentations pour apprécier la vulnérabilité systémique? Les indicateurs construits par chaque composante (aléas, enjeux, gestion, représentations) seront ensuite agrégés pour former des indices composites de vulnérabilité transversaux aux composantes, et aboutir à un indice global de vulnérabilité systémique par territoire, dont la pertinence sera discutée. La seconde question à traiter dans la suite du projet concerne le mode de représentation de l'évolution, sur plusieurs années, des indicateurs et des indices de vulnérabilité, sous la forme d'une interface web utilisable par les gestionnaires (Marcel, 2017). Cette représentation devra considérer de nombreux éléments : l'échelle spatiale la plus pertinente, des résultats à différents niveaux d'agrégation des indicateurs en indices de vulnérabilité, et la variation annuelle des résultats.

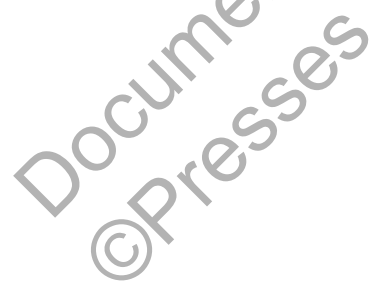

3. Un nouveau projet OSIRISC+ (2017-2019), soutenu par la DREAL Bretagne, va permettre ces tests sur plusieurs communes du golfe du Morbihan.

\section{Remerciements}

Les auteurs remercient pleinement les participants à la construction de cette recherche qui est avant tout l'aboutissement du travail d'un groupe pluridisciplinaire, intégrant l'ensemble des membres des quatre composantes du projet OSIRSC, financé par la Fondation de France. Une attention particulière est également portée aux gestionnaires et élus constituant le comité de suivi du projet, personnes ressources dans la construction des indicateurs, et plus largement de l'observatoire de la vulnérabilité aux risques côtiers. Enfin, les auteurs tiennent à remercier les relecteurs et le comité de rédaction de la revue Norois pour la pertinence de leurs remarques.

\section{Bibliographie}

André C., 2013. Analyse des dommages liés aux submersions marines et évaluation des coûts induits aux habitations à partir de données d'assurance : perspectives apportées par les tempêtes Johanna (2008) et Xynhtia (2010). Thèse de doctorat, Université de Bretagne Occidentale, 328 p.

Bernon N., Mallet C., Belon R., avec la collaboration de Hoareau A., Bulteau T., Garnier C., 2016. Caractérisation de l'aléa recul du trait de côte sur le littoral de la côte aquitaine aux horizons 2025 et 2050. Rapport final, BRGM/ RP66277-FR, 99 p.

Boulanger P.M., 2004. Les indicateurs de développement durable : un défi scientifique, un enjeu démocratique, Les séminaires de l'Iddri, n ${ }^{\circ} 12,24 \mathrm{p}$.

Bвоокs N., 2003. Vulnerability, Risk and Adaptation: A Conceptual Framework. Tyndall Centre for Climate Change Research, $\mathrm{n}^{\circ}$ 38, $16 \mathrm{p}$.

Chaumillon E., Bertin X., Fortunato A.B., Bajo M., Schneider J.L., Dezileau L., Walsh J.P., Michelot A., Chauveau E., Creach A., Henaff A., Sauzeau T., Waeles B., Gervais B., Jan G., Baumann J., Breilh J.F., Pedreros R., 2017. Storm-induced marine flooding: Lessons from a multidisciplinary approach. Earth-Science Reviews, Elsevier, vol. 165, p. 151-184. DOI : 10.1016/j.earscirev.2016.12.005

Chauveau, E., Chadenas C., Comentale B., Pottier P., Blanloeil A., Feuillet T., Mercier D., Pourinet L., Rollo N., Tillier I., Trouillet B., 2011. Xynthia : leçons d'une catastrophe. Cybergeo : European Journal of Geography [En Ligne]. DOI : 10.4000/cybergeo. 23763

Chevillot-Miot E., Mercier D., 2014. La vulnérabilité face au risque de submersion marine : exposition et sensibilité des communes littorales de la région Pays de la Loire (France). VertigO [En Ligne], n² 2, vol. 14. DOI : 10.4000/ vertigo. 15110 
CESER de L'AtLANTique, 2015. Submersion marine et érosion côtière : connaitre, prévenir et gérer les risques naturels littoraux sur la façade atlantique. Rapport CESER de l'Atlantique, $76 \mathrm{p}$.

CETMEF, CETE Méditerranée, CETE de l’Ouest, 2009. Vulnérabilité du territoire National aux risques littoraux. Rapport CETMEF/DELCE, 163 p.

Daubord C., 2014. Caractérisation de 7 évènements de tempête de l'automne-hiver 2013-2014 à partir des données disponibles au SHOM. Rapport du SHOM no 001/2014, 37 p.

Direction Départementale des Territoires et de la Mer Finistère (DDTM 29), 2016. Plan de Prévention des risques littoraux «Ouest Odet ». Approuvé le 12 juillet 2016.

Deboudt P., 2010. Vers la mise en œuvre d'une action collective pour gérer les risques naturels littoraux en France métropolitaine. Cybergeo : European Journal of Geography [En Ligne]. DOI: 10.4000/cybergeo.22964

DEDUCE CONSORTIUM, 2006. Mettre en oeuvre une approche basée sur les indicateurs pour évaluer le développement durable sur le littoral. Guide d'utilisation des indicateurs, $70 \mathrm{p}$.

D’Ercole R., Thouret J.-C., Dollfus O., Asté J.-P., 1994. Les vulnérabilités des sociétés et des espaces urbanisés : concepts, typologie, modes d'analyse. Revue de Géographie Alpine/Journal of Alpine Research, $\mathrm{n}^{\circ}$ 4, vol. 82, p. 87-96. DOI : 10.3406/rga.1994.3776

D'Ercole R., 1996. De l'évaluation à la gestion des risques : 1a vulnérabilité en question. Pangea, no 25 , p. 5-12.

D'Ercole R., 1998. Approches de la vulnérabilité et perspectives pour une meilleure logique de réduction dés risques. Pangea, n 29/30, p. 20-28.

Direction Générale de la Prévention des Risques (ĐGPR), 2017. Cahier des charges PAPI 3, MEEM,61 p.

Direction Générale de la Prévention des Risques (DGPR), 2014. Guide méthodologique : plan de prévention des risques littoraux. MEDDE, 169 p.

DREAL Pays de la Loire, 2012. La tempête Xynthia du 28 février 2010, retour dexperience en Loire Atlantique et en Vendée, volet hydraulique et ouvrages de protection. Rapport de la DREAL Pays de la Loire, 78 p.

Dupont C., 2016. Elaboration d'indicateurs pour l'évaluation de la mise en cuvre de processus d'intégration des politiques maritimes en Méditerranée. Mémoire de Master Expertise et Gestion de l'Environnement Littoral, Université de Bretagne Occidentale, $80 \mathrm{p}$.

Gallopin G.-C., 1997. Indicators and Their Use: Information for Decision-making, in Moldan B., Bilharz S. (eds.), Sustainability Indicators: A Report on the Project on Indicators of Sustainable Development. Scientific Committee on Problems of the Environnement. p. 13-27.

GIP Littoral Aquitain, 2012. Stratégie régionale de gestion de la bande côtière : sensibilité régionale à l'érosion côtière. $180 \mathrm{p}$.

Hellequin A.-P., Flanquart H., Meur-Férec C., Rulleau B., 2013. Perceptions du risque de submersion marine par la population du littoral languedocien : contribution à l'analyse de la vulnérabilité côtière, Natures Sciences Sociétés, n 4 , vol. 21, p. 385-399. DOI : 10.1051/nss/2014002

Hénaff A. (dir.), Philippe M. (coord.), 2014. Gestion des risques d'érosion et de submersion marine, guide méthodologique. Projet Cocorisco. 156 p.

Lafitte A., Denis J., Henocque Y., Andral B., Boissery P., 2012. Elaboration et test d'indicateurs de suivi des initiatives locales de gestion intégrée du littoral méditerranéen, Revue Paralia, vol. 5, p. 4.1-4.12. DOI : 10.5150/revue-paralia.2012.004

LENDRE S., 2016. Vers des indicateurs de suivi de la vulnérabilité côtière aux risques d'érosion et de submersion. Mémoire de Master Expertise et Gestion de l'Environnement Littoral, Université de Bretagne Occidentale, 59 p.

Leone F., Vinet F., 2006. La vulnérabilité, un concept fondamental au cœur des méthodes d'évaluation des risques naturels, in LeONe F., Vinet F. (dir.), La vulnérabilité des sociétés et des territoires face aux menaces naturelles : analyse géographique. Géorisque, no 1, p. 9-25.

LEONE F., 2007. Caractérisation des vulnérabilités aux catastrophes "naturelles : contribution à une évaluation géographique multirisque lmouvements de terrain, séismes, tsunamis, éruptions volcaniques, cyclones). Habilitation à diriger des recherchès, Géographie, Montpellier 3, Université Paul Valéry, $245 \mathrm{p}$.

MarcelO., 2017. Prototypage d'une interface WEB-SIG pour - l'Observatoire Intégré des Risques Côtiers. Mémoire de Master Carthagéo, École Nationale des Sciences Géographiques, $64 \mathrm{p}$.

Metzger P., D'Ercole R., 2011. Les risques en milieu urbain : éléments de réflexion, EchoGéo [En ligne], nº 18. DOI : 10.4000/echogeo. 12640

Meur-Férec C. (coord.), Deboudt P., Deldrève V., Flanquart H., Hellequin A.P., Herbert V., Longuepée J., Morel V., 2004. La vulnérabilité des territoires côtiers : évaluation, enjeux et politiques publiques. Programme National Environnement Côtier, rapport final, $56 \mathrm{p}$.

Meur-Férec C., 2006. De la dynamique naturelle à la gestion intégrée de l'espace littoral : un itinéraire de géographe. Habilitation à diriger des recherches, Géographie, Université de Nantes, 248 p. [https://tel.archives-ouvertes.fr/tel00167784/document].

Meur-Férec C., Deboudt P., Morel V., 2008. Coastal Risks in France: An Integrated Method for Evaluating Vulnerability, Journal of Coastal Research, vol. 24, p. 178-189.

Meur-Férec C., Rabuteau Y., 2014. Plonevez-les-Flots : un territoire fictif pour souligner les dilemmes des élus locaux face à la gestion des risques côtiers, L'Espace Géographique, $n^{\circ} 1$, vol. 43, p. 17-33. DOI : 10.3917/eg.431.0018

Michel-Guillou E., Meur-Férec C., 2016 . Representations of coastal risks (erosion and marine flooding) among inhabitants of at-risk municipalities, Journal of Risk Research, $\mathrm{n}^{\circ}$ 6, vol. 20, p. 776-799. DOI : 10.1080/13669877.2015.1119181

Mineo-Kleiner L., 2017. L'option de la relocalisation des activités et des biens face aux risques côtiers : stratégies et enjeux 
territoriaux en France et au Québec. Thèse de doctorat en Géographie, Université de Bretagne occidentale, 350 p.

Miossec A. (dir.), 2012. Dictionnaire de la mer et des côtes, Rennes, Presse universitaire de Rennes, $552 \mathrm{p}$.

Perherin C., 2017. La concertation lors de la cartographie des aléas littoraux dans les Plans de Prévention des Risques : enjeu majeur de prévention. Thèse de doctorat en Géographie Université de Bretagne Occidentale, 377 p.

Quillet E., 2017. Élaborer un suivi de la gestion des risques côtiers d'érosion - submersion à partir d'indicateurs : application dans le cadre du projet OSIRISC. Mémoire de Master Géographie et Aménagement des Espaces Maritimes, Université de Nantes, 112 p.

Reghezza M., 2006. La vulnérabilité : un concept problématique, in LeONe F., Vinet F. (dir.), La vulnérabilité des sociétés et des territoires face aux menaces naturelles: analyse géographique. Géorisque, nº 1, p. 9-25.
Rey-Valette H., Carbonnel P., Roussel S., Richard A., 2006. L'apport de la gestion intégrée des zones côtières (GIZC) à la gestion de l'érosion côtière : intérêt et exemple en Méditerranée française, VertigO [en ligne], nº 3, vol. 7. DOI : 10.4000/vertigo. 2529

Rey-Valette H., Rulleau B., Hellequin A.-P., MeurFérec C., Flanquart H., 2014. Second-home owners and sea-level rise: the case of the Languedoc-Roussillon region (France), Journal of Policy Research in Tourism, Leisure and Events, $\mathrm{n}^{\circ}$ 1, vol. 7, p. 32-47. DOI : 10.1080/19407963.2014.942734

SIVOM Combrit - Île Tudy, 2011. Projet de Programme d'Actions de Prévention des Inondations (PAPI). 128 p.

Veyret Y. (dir.), 2007. Dictionnaire de l'environnement, Paris, Armand Colin, 404 p.

Wisner B., O’Keefe P., Westgate K., 1976. Taking the Naturalness out of Natural Disasters. Nature, n² 260, p. 566-567. 


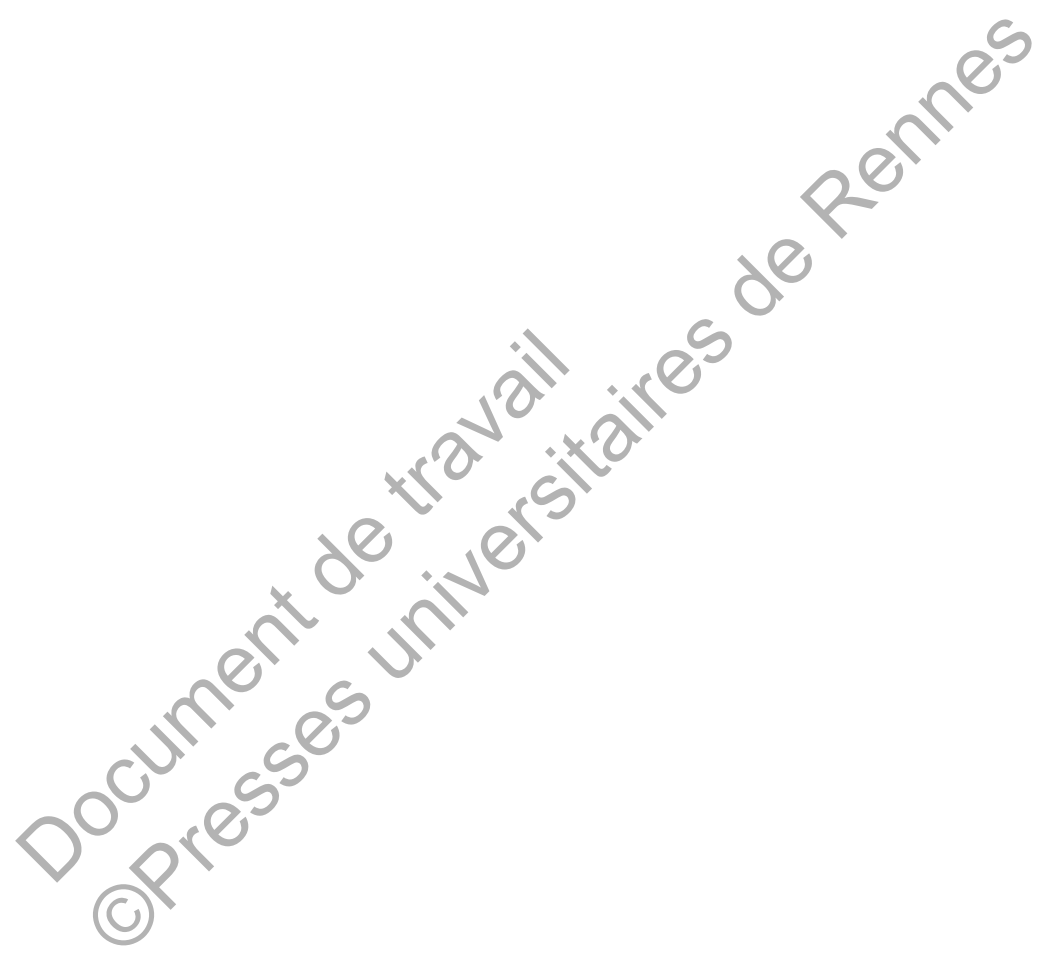

